\title{
Research on the early warning method of transformer fire fault in ocean booster station based on fault tree model
}

\author{
Fuqiang Zhang ${ }^{1}$, Zerong Yang ${ }^{1}$, Ting $\mathrm{Li}^{1}$, Yue $\mathrm{Jia}^{1}$, Dan Zhou ${ }^{2 *}$ and Luyao Xie ${ }^{2}$ \\ ${ }^{1}$ Guodian Power Zhejiang Zhoushan Offshore Wind Power Development Co., Ltd, Zhoushan, Zhejiang, 316000, China \\ ${ }^{2}$ College of Information Engineering, Zhejiang University of Technology, Hangzhou, Zhejiang, 310014, China
}

\begin{abstract}
As the heart of the sea booster station, the transformer has an extremely important position. When the transformer has various faults, it is very easy to cause a fire accident, so the safe operation of the transformer is very important. First, use the fault tree analysis method to construct a transformer fire fault tree evaluation model for different types of fire fault events during the operation of the transformer, then through the calculation of the fire failure probability, the number of measuring points of the composite fire detector is obtained and the measuring points are reasonably arranged to detect the operation status of the transformer in real time. Therefore, it is of great significance to ensure the safe and reliable operation of offshore booster station by improving the accuracy of transformer fire warning.
\end{abstract}

\section{Introduction}

Sea booster stations are generally far away from the land, and most of them are designed in accordance with the "unattended" operation mode. When the transformer has a fire failure, it will bring significant economic losses. In order to avoid losses and false alarms, the fault tree model is used to analyze the cause of fire caused by transformer failure, and the installation layout of the composite fire detector's measuring points is optimized to improve the accuracy of fire warning.

A reasonable transformer fire fault model is the basis of transformer state detection. Literature [1] studies the transient behavior of transformer winding and determines the location of winding faults; Literature [2] diagnoses transformer faults based on the analysis of dissolved gas in oil; Literature [3] uses the method of searching the coil to detect the fault of the winding, and classify and locate the fault. Literature [4] uses the method of point impedance test of the necessary physical and electrical parameters of the transformer to diagnose the fault of the active part of the transformer; Literature [5] accurately calculates transformer winding temperature and seeks the quantitative relationship between winding temperature and transformer failure rate. The above-mentioned documents mainly focus on the study of the fire fault of a single part of the transformer, The above-mentioned documents mainly focus on the study of the fire fault of a single part of the transformer, did not fully consider its fire failure.

For this reason, this article first established a transformer fire fault tree model based on the need for transformer fire fault diagnosis in the sea booster station. Secondly, according to the fire fault tree model, the probability of fire faults in various parts of the transformer is calculated and the composite fire detectors are installed reasonably. Finally, the effectiveness of the proposed transformer fire fault tree model is verified through the analysis of calculation examples.

\section{Analysis of transformer fire fault tree}

\subsection{Overview of fault tree analysis}

Fault tree analysis (FTA) is a top-down deductive failure analysis method that uses Boolean logic to combine lowlevel events to analyze undesirable states in the system.

The minimum cut set is the basic concept of fault tree analysis, which expresses the most necessary and least combination of bottom events that cause top events to occur.

This article assumes that a transformer fault can consist of $k$ basic events, namely $x_{1}, x_{2}, \ldots, x_{k}$, the probabilities are $P\left(x_{1}\right), P\left(x_{2}\right), \ldots, P\left(x_{k}\right)$ the minimum cut set of the fault tree is $C_{1}, C_{2}, \ldots, C_{i}, \ldots, C_{n}$ therefore, the total failure rate of the transformer $P(T)$ is:

$$
P(T)=P\left(\stackrel{a}{i=1}_{i}^{n} C_{i}\right), i=1,2, \text { 吹, , } n
$$

According to the probability plus the statute:

$$
\left.P(T)=P\left(\sum_{i=1}^{n} C_{i}\right)-\sum_{i k j}^{n} \sum P\left(C_{i} C_{j}\right)-\ldots(-1)^{m} \mathrm{P}_{\left(C_{1}\right.} \ldots C_{2}\right)
$$

\footnotetext{
"Corresponding author's e-mail: zhoudan@zjut.edu.cn
} 
When the fault tree is large, there are many minimum cut sets, so in engineering calculations, the upper bound is usually used to approximate the probability of transformer failure, namely:

$$
P(T)=\sum_{i=1}^{n} P\left(C_{i}\right)
$$

\subsection{Transformer fire fault tree model}

Building the transformer fire fault tree model is divided into the following stages:

(1) Top event: Located at the top of the fault tree, it is the least wanted event in the entire device, so it is the output terminal of the logic gate. The top event in this article is a transformer failure.
(2) Intermediate event: Located between the top event and the bottom event, it is the direct factor and cause of the possible occurrence of the top event. The intermediate events in this article are winding failure, iron core failure, bushing failure, tap changer failure, lead failure and other failures.

(3) Bottom event: Located at the end of the fault tree, it is the basic cause of the top event.

(4) The intermediate event and the bottom event are connected through the "or" relationship in the logic gate to form the fault tree model of the transformer.

According to the statistical analysis of transformer fire fault data, the transformer fire fault tree model is shown in figure 1, the symbol description of the fault tree model and the fire fault probability of the transformer bottom event are shown in table 1 and table 2 respectively.

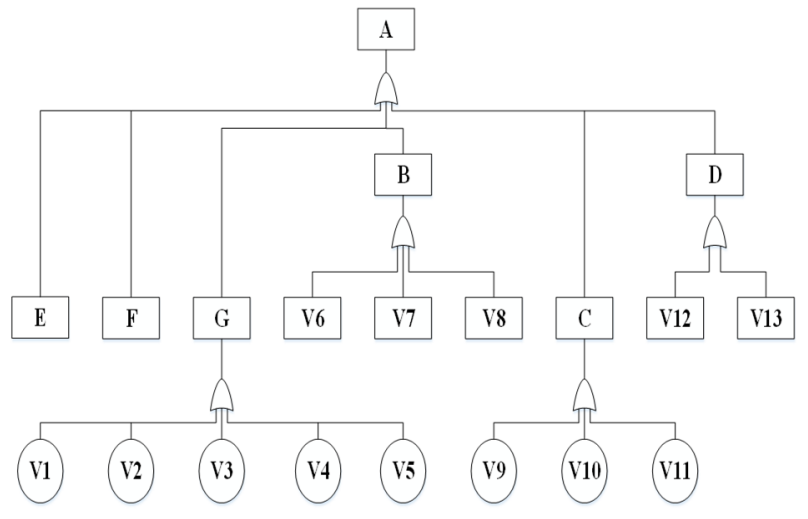

Figure 1. Transformer fault tree mode.

Table 1. Symbol description of fault tree model.

\begin{tabular}{cccc}
\hline Symbol & Symbol description & Symbol & Symbol description \\
A & Transformer failure & V4 & Poor maintenance process \\
B & Core failure & V5 & Poor production process \\
C & Bushing failure & V6 & Core multi-point grounding \\
D & Tap changer failure & V7 & Reduced resistance to ground \\
E & Lead failure & V8 & Local overheating \\
F & Other failures & V9 & Poor sealing \\
G & Winding failure & V10 & mechanical injury \\
V1 & Weak insulation & V11 & Bushing overheated \\
V2 & Atmospheric over-voltage & V12 & Insufficient contact pressure \\
V3 & Operating over-voltage & V13 & Contact point stains \\
\hline
\end{tabular}

\subsection{Model calculation of transformer fire fault tree}

According to the upward method, the minimum cut set of the transformer fire fault tree model can be expressed as: $C=\{\mathrm{V} 1, \mathrm{~V} 2, \mathrm{~V} 3, \mathrm{~V} 4, \mathrm{~V} 5, \mathrm{~V} 6, \mathrm{~V} 7, \mathrm{~V} 8, \mathrm{~V} 9, \mathrm{~V} 10, \mathrm{~V} 11, \mathrm{~V} 12, \mathrm{~V} 13, \mathrm{E}, \mathrm{F}\}$

From formula (3), the probability of transformer fire failure can be calculated as:

$$
P(T)=\sum_{i=1}^{n} P\left(C_{i}\right)=\sum_{i=1}^{15} P\left(C_{i}\right)
$$

From the same principle of formula (3), the probability of fire failure in transformer winding, iron core, bushing, tap switch and lead wire can be calculated as follows:

$$
\begin{gathered}
P_{1}(T)=\sum_{i=1}^{n} P\left(C_{i}\right)=\sum_{i=1}^{5} P\left(C_{i}\right) \\
P_{2}(T)=\sum_{i=6}^{n} P\left(C_{i}\right)=\sum_{i=6}^{8} P\left(C_{i}\right) \\
P_{3}(T)=\sum_{i=9}^{n} P\left(C_{i}\right)=\sum_{i=9}^{11} P\left(C_{i}\right)
\end{gathered}
$$




$$
\begin{gathered}
P_{4}(T)=\sum_{i=12}^{n} P\left(C_{i}\right)=\sum_{i=12}^{13} P\left(C_{i}\right) \\
P_{5}(T)=\mathrm{P} \quad\left(C_{14}\right)
\end{gathered}
$$

According to formula (5 9), the probability of fire failure in various parts of the entire transformer equipment can be calculated. On this basis, this paper arranges the detection device by comparing the weakness of the fire failure.

\section{Transformer fire detection method based on fire fault analysis}

Figure 2 shows the parts of transformers in the sea booster station that are prone to fire.

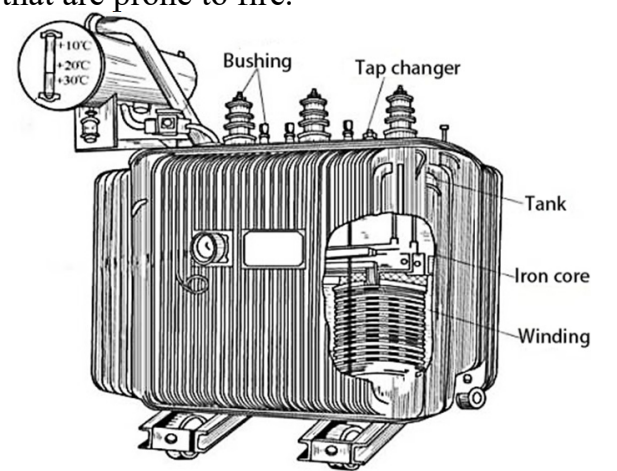

Figure 2. Diagram of transformer fire fault location.

The composite fire detector is composed of carbon monoxide, photoelectric smoke and temperature sensor, and uses a microprocessor to perform composite detection processing on the transformer fire fault part shown in figure 2 .

The number of equidistant measuring points between the winding and the iron core can be determined according to formula (10), namely

$$
\lambda_{1}=\frac{P_{1}(T)+P_{2}(T)}{P(T)} \times H
$$

where $\lambda_{1}$ represents the number of measuring points installed at the winding and iron core parts; $H$ represents the total installed number of composite fire detectors;
$P(T)$ represents the probability of transformer fire failure; $P_{1}(T)$ represents the probability of winding fire failure; $P_{2}(T)$ represents the probability of iron core fire failure.

$$
\lambda_{2}=\frac{P_{3}(T)}{P(T)} \times H
$$

where $\lambda_{2}$ represents the number of measuring points installed in the casing; $P_{3}(T)$ represents the probability of fire failure in the casing.

The number of equally spaced measuring points at the tap changer can be determined according to formula (12), namely

$$
\lambda_{3}=\frac{P_{4}(T)}{P(T)} \times H
$$

where $\lambda_{3}$ represents the number of measuring points installed at the tap changer; $P_{4}(T)$ represents the probability of fire failure of the tap changer.

The number of equidistant measuring points at the lead position can be determined according to formula (13), namely

$$
\lambda_{4}=\frac{P_{5}(T)}{P(T)} \times H
$$

where $\lambda_{4}$ represents the number of measuring points installed at the lead position; $P_{5}(T)$ represents the probability of fire failure of the lead.

\section{Case study}

\subsection{Parameter settings}

The calculation examples in this section mainly analyze the operability of arranging the measuring points of the composite fire detector based on the probability of the proposed transformer fire fault tree model. The total number of selected measuring points is 16 .

The specific parameter settings for the probability of a transformer fire fault location are shown in table 2.

Table 2. Parameter setting of fire failure probability of transformer bottom event.

\begin{tabular}{cccc}
\hline Fault location & Event symbol & Basic event & Probability of fire \\
& V1 & Weak insulation & 0.05167 \\
& V2 & Atmospheric over-voltage & 0.0372 \\
Winding & V3 & Operating over-voltage & 0.0248 \\
& V4 & Poor maintenance process & 0.01653 \\
& & & \\
& V5 & Poor production process & 0.02479 \\
Iron core & V6 & Multi-point grounding of iron core & 0.03573 \\
& V7 & Reduced resistance to ground & 0.00715 \\
Bushing & V9 & Local overheating & 0.01072 \\
& V10 & Poor sealing & 0.01679 \\
Tap changer & V11 & mechanical injury & 0.01509 \\
& V12 & Casing overheated & 0.01844 \\
& V13 & Insufficient contact pressure & 0.00613 \\
& & Contact point stains & 0.01226 \\
\hline
\end{tabular}




\begin{tabular}{cccc}
\hline lead & E & Lead failure & 0.0625 \\
other & $\mathrm{F}$ & Other failures & 0.0745 \\
\hline
\end{tabular}

\subsection{Result analysis}

According to formula (4 9) and combine the parameters in table 2 , and the results obtained are shown in table 3.

Table 3. Fire failure probability of transformer sub-system.

\begin{tabular}{cccccccc}
\hline Failure event & Transformer & Winding & Iron core & Bushing & Tap changer & Lead & Other \\
Fire failure rate & 0.4143 & 0.1550 & 0.0536 & 0.0503 & 0.0184 & 0.0625 & 0.0745 \\
\hline
\end{tabular}

According to formula (10 13), the number of and other parts can be calculated in turn, as shown in table installation points of composite fire detectors in winding 4.

Table 4. Number of measuring points installed.

\begin{tabular}{cccccc}
\hline Transformer part & Winding, iron core & Bushing & Tap changer & Lead & Other \\
Installed quantity & 8 & 2 & 1 & 2 & 3 \\
\hline
\end{tabular}

By optimizing the installation of measuring points, the accuracy of the transformer fire warning in the sea booster station is improved, and false alarms are avoided.

\section{Conclusion}

Aiming at the hazard of the main transformer fire failure in the sea booster station, this article first analyzes the cause of the transformer fire failure. On this basis, a transformer fire fault tree model was established based on the fault tree theory, and the probability of each fire fault was calculated. Secondly, the distribution of composite fire detectors is reasonably arranged based on the probability of fire failure. Finally, simulations verify that the method proposed in this paper to use composite fire detectors based on the characteristics of fire faults in different parts of the transformer can effectively improve the accuracy of transformer fire warning and ensure the safe and reliable operation of the sea pressure station in the "unattended" operation management.

\section{References}

1. NOSRATIAN AHOUR J, SEYEDTABAII S. (2017) Determination and localisation of turn-to-turn fault in transformer winding using frequency response analysis. IET Science, 12: 291-300.

2. SINGH S,BANDYOPADHYAY M. (2010)Dissolved gas analysis technique for incipient fault diagnosis in power transformers. IEEE Electrical Insulation Magazine, 26: 41-46.

3. VENIKAR P A, BALLAL M S. (2017)Search Coil Based Online Diagnostics of Transformer Internal Faults. IEEE Transactions on Power Delivery, 32: 2520-2529.

4. PHAM D A K, PHAM T M T. (2013)A new method for purposes of failure diagnostics and FRA interpretation applicable to power transformers. IEEE Transactions on Dielectrics and Electrical Insulation, 20: 2026-2034.

5. FERNANDEZ F O, ORTIZ A. (2017)Transformer health indices calculation considering hot-spot temperature and load index. IEEE Electrical Insulation Magazine, 33: 35-43. 